\title{
EVOLUTION OF THE MILLIARCSECOND POLARIZATION STRUCTURE OF THE SUPERLUMINAL QUASAR 3 C345
}

\author{
J. F. C. Wardle, D. H. Roberts, L. F. Brown, and D. C. Gabuzda \\ Department of Physics \\ Brandeis University \\ Waltham, MA 02254 USA
}

\begin{abstract}
The $\lambda 6 \mathrm{~cm}$ milliarcsecond polarization structure of $3 \mathrm{C} 345$ has been determined at three epochs between December 1981 and March 1984. The knots C2, C3, and $\mathrm{C} 4$ all showed changes as they moved away from the core, which remained virtually unpolarized.
\end{abstract}

\section{INTRODUCTION}

We have embarked on a program to study the the structure and evolution of the polarization of $3 \mathrm{C} 345$ (and other sources) at milliarcsecond resolution. A first epoch (1981.92) map of 3C345 was presented by Wardle et al. 1986. Here we show preliminary maps made from data taken in December 1983 and March 1984, and give the first report of the evolution of the milliarcsecond polarization structure.

\section{OBSERVATIONS AND DATA PROCESSING}

Polarization-sensitive $\lambda 6 \mathrm{~cm}$ observations were carried out in December 1983 and March 1984 with the Bonn (1984 only), Haystack, Green Bank, Fort Davis, and Owens Valley antennas and the phased-up VLA, using the Mark III data recording system. Maps of the total and polarized intensity were made as described by Roberts et al. (1984) and Wardle et al. (1986).

\section{RESULTS}

Maps of the $I$ and $P$ distributions of 3C345 in December 1983 and March 1984 are shown in Figures 1 and 2. The total intensity maps are in good agreement with those of Biretta, Moore, and Cohen (1986). The evolution of the polarization of the core and knots of $3 \mathrm{C} 345$, derived from summing CLEAN components or by model fitting, is summarized in Table 1. The polarization of the core of $3 \mathrm{C} 345$ is very small $(\approx 1 \%)$ at each epoch, requiring a very efficient depolarization mechanism (if the radiation is from the synchrotron process in an ordered magnetic field). The knots are moderately polarized, and as they move away from the core their electric vectors are seen to change. This may be due to changing orientation of the magnetic field, a changing Faraday rotation, or changing relativistic aberration. Additional observations of $3 \mathrm{C} 345$ at $\lambda \lambda 2.8$ and $6 \mathrm{~cm}$, which can decide among these possibilities, are being reduced. 
We thank the staffs of the US VLBI Network observatories and of the Haystack Mark III Processor for their help. Financial support was provided by the NSF under grants AST82-13991 and AST-84-18636 (JFCW) and AST-83-15945 and AST-85-19529 (DHR).

\section{REFERENCES}

Biretta, J. A., Moore, R. L., and Cohen, M. H. 1986, Astrophys. J., 308, 93-109.

Roberts, D. H., et al., 1984, in VLBI and Compact Radio Sources, ed. R. Fanti, K. Kellermann, and G. Setti (Dordrecht: Reidel), 35-38.

Wardle, J. F. C., Roberts, D. H., Potash, R. I., and Rogers, A. E. E. 1986, Astrophys. J. (Letters), 304, L1-L4.
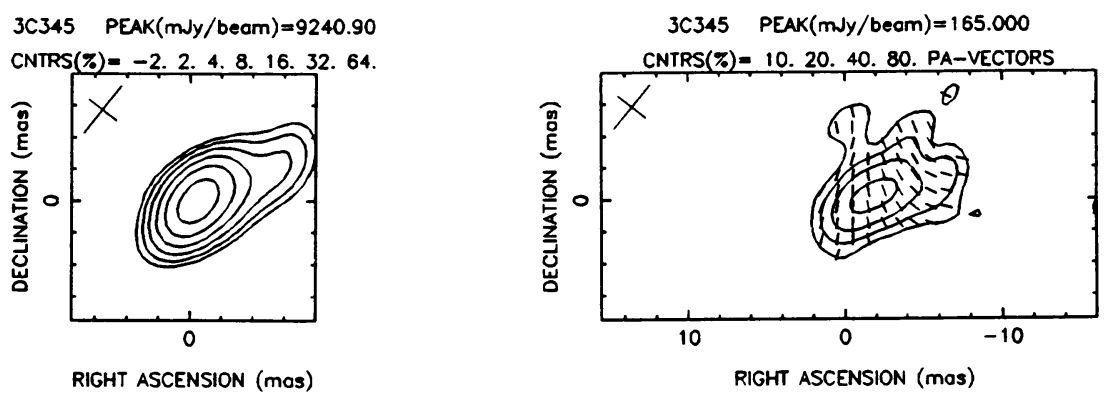

Figure 1: 3C345, epoch 1983.93 (one tick mark is 2 mas). (a) Total intensity. (b) Polarized intensity, with contours of polarized flux and electric vector position angles indicated by the line segments.
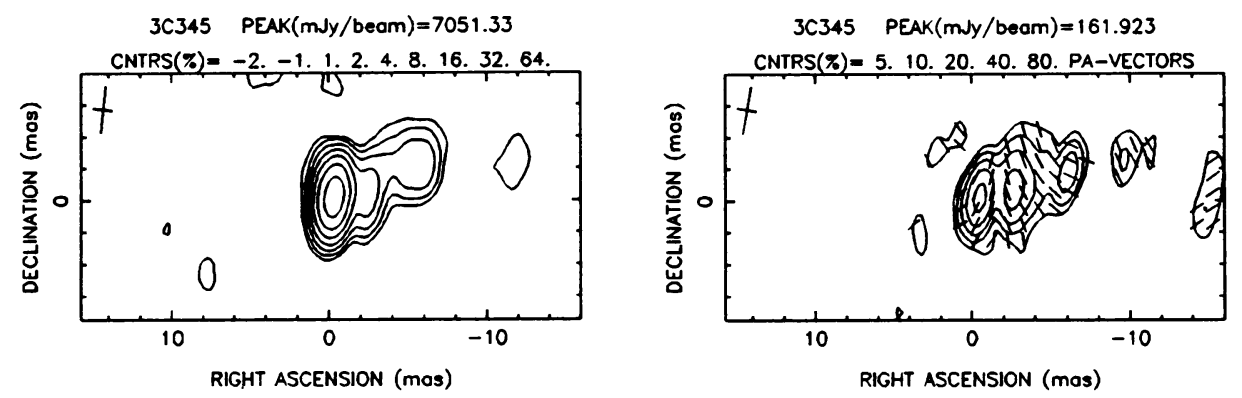

Figure 2: 3C345, epoch 1984.23. Same parts as Figure 1.

Table 1: Evolution of the Polarization of the Components of 3C345

\begin{tabular}{ccccccc}
\hline & \multicolumn{2}{c}{1981.92} & \multicolumn{2}{c}{1983.93} & \multicolumn{2}{c}{1984.23} \\
\hline Component & $m(\%)$ & $\chi(\mathrm{deg})$ & $m(\%)$ & $\chi(\mathrm{deg})$ & $m(\%)$ & $\chi(\mathrm{deg})$ \\
\hline D & $<1$ & $\ldots$ & $\sim 1^{a}$ & $? ?$ & $\sim 1^{b}$ & -72 \\
C4 & $\ldots$ & $\ldots$ & $\ldots$ & $\ldots$ & 4. & -19 \\
C3 & 11. & +22. & 13. & +34. & 14. & +28. \\
C2 & 6. & +83. & 9. & +74. & 6. & +61. \\
\hline
\end{tabular}

${ }^{a}$ Due in part to $\mathrm{C} 4$.

${ }^{b}$ Due in part to $\mathrm{C} 5$. 